\title{
PERCEPÇÃO E USO DOS ESPAÇOS AGRÍCOLAS INTRA-URBANOS
}

\author{
intra-urban agricultural spaces: an approach from the environment and behavior studies
}

\author{
Daniele Tubino Pante de Souza ${ }^{1}$ \\ Antônio Tarcísio da Luz Reis ${ }^{2}$
}

$a_{a} \boldsymbol{a a}_{a}$

\begin{abstract}
Resumo
Este artigo discute os espaços de agricultura intra-urbana a partir da abordagem da área de estudos ambiente-comportamento. Embora os espaços destinados ao cultivo de alimentos estejam cada vez mais presentes nas cidades, as relações entre esses e os seus usuários necessitam de uma compreensão mais aprofundada. É adotado como foco de estudo as hortas comunitárias e discutido o uso e a percepção de seus usuários diretos e indiretos. As hortas comunitárias contribuem para o bem-estar psicológico de usuários diretos e são percebidas como espaços de socialização. Desse modo, o estudo da percepção e do uso dos espaços urbanos de cultivo mostra-se importante na medida em que esses espaços desempenham um papel no atendimento das necessidades fisiológicas, psicológicas e sociais dos cidadãos..
\end{abstract}

Palavras-chave: Agricultura urbana, Ambiente-comportamento, Hortas comunitárias.

\begin{abstract}
This article aims to discuss the areas of urban agriculture on the light of the field of environment-behavior studies. Although the areas intended for growing food are increasingly present in the cities, the relations between such areas and their users still need a better understanding. This work has adopted the community gardens as a focus of study and discuss the use and the perception of direct and indirect users of these spaces. Regarding to the active interaction with nature, community gardens contribute to psychological well-being of direct users. In addition, they are perceived as spaces for socialization and stimulate the generation of sense of community. Thus, the perception and the use of areas for growing food in cities should be considered as an important part in the role played by such spaces in meeting the physiological, psychological and social needs of citizens.
\end{abstract}

Key words: Urban agriculture, Environment and behavior, Community gardens

\begin{abstract}
Resumen
Este artículo analiza los espacios de la agricultura intra-urbana a la luz de los estudios de ambiente-comportamiento. Aunque los espacios destinados a cultivar alimentos están cada vez más presente en las ciudades, las relaciones entre estos y sus usuarios requieren una comprensión más profunda. Se adoptó como foco de estudio huertas comunitárias, y fue discutido el uso y la percepción de los usuarios directos e indirectos de estos lugares. Las huertas comunitarias contribuyen al bienestar psicológico de los usuarios directos y son percibidas como espacios de socialización. De esta forma, el estudio de la percepción y el uso de la agricultura urbana es importante en la medida en que estas áreas desempeñan un papel en la satisfacción de las necesidades fisiológicas, psicológicas y sociales de los ciudadanos.
\end{abstract}

Palabras claves: agricultura urbana, ambiente-comportamiento, huertas comunitarias

(1) Mestre em Engenharia Civil da Universidade Federal do Rio Grande do Sul - Av. Osvaldo Aranha 99, $3^{\circ}$ andar, CEP: 90050170, Porto Alegre (RS), Brasil, Tel./Fax.: (+55 51) 33083518 - danieletubino@gmail.com

(2) Bolsista Produtividade CNPq, Prof. Dr. Programa de Pós-Graduação em Planejamento Urbano e Regional da Universidade Federal do Rio Grande do Sul - Rua Sarmento Leite, 320, CEP: 90050-170, Porto Alegre (RS), Brasil, Tel.: (+55 51) 33083145 - tarcisio@orion.ufrgs.br

\section{aaAaa}




\section{INTRODUÇÃO}

A agricultura urbana vem sendo praticada de diversas formas nas cidades, ao longo do tempo. Hortas comunitárias, hortas escolares e hortas domésticas representam alguns exemplos. Esses espaços estão presentes nas cidades por diversos motivos, como por exemplo, para subsistência de populações de baixa renda, para o fortalecimento das redes sociais em comunidades ou para a realização de educação ambiental (REYNOLDS, 2010). Contudo, nos últimos 150 anos, a incorporação de atividades agrícolas em meio urbano não se revelou como um foco de grande interesse público (Ibid). Os períodos em que se percebeu um maior incentivo à produção de alimentos nas cidades estiveram, geralmente, associados com graves crises econômicas e sociais, decorrentes, por exemplo, das Guerras Mundiais ou de recessões econômicas, tal como a recessão americana de 1929 (DEELSTRA; GIRARDET, 2000). Nesses períodos, o cultivo de alimentos em grandes cidades da América do Norte e da Europa, por meio de hortas comunitárias, foi essencial para a subsistência das populações urbanas (LAWSON, 2004).

A partir da década de 80 ressurgiu um grande interesse em relação à agricultura urbana (MAXWEEL, 2001). De acordo com Deelstra e Girardet (2000), essa revalorização de espaços produtivos pode ser atribuída tanto à intensificação das discussões sobre os impactos ambientais gerados pelo modelo de desenvolvimento, quanto ao aumento das taxas de desemprego nas grandes cidades. Desse modo, tem-se observado nas últimas décadas, um número crescente de iniciativas para promover a integração de locais de produção de alimentos às cidades (REYNOLDS, 2010).

A incorporação de atividades de agricultura em áreas urbanas pode gerar benefícios ambientais e sócio-econômicos. Sob o ponto de vista ambiental, a agricultura urbana representa uma estratégia para a redução dos impactos gerados pelas cidades e pela atual forma de produção de alimentos (p. ex. DEELSTRA; GIRARDET, 2000; MOUGEOT, 2000, SMIT; NASR, 1992). Smit e Nasr (1992) referem-se à agricultura urbana como uma atividade que viabiliza processos de ciclos fechados nas cidades. Como parte desses processos, os resíduos urbanos podem ser compostados e utilizados como recurso nos processos de produção de alimentos. Sob o enfoque sócio-econômico, a agricultura urbana promove a geração de renda e de empregos, resultando em maior vitalidade da economia urbana (MOUGEOT, 2000). A atividade está também relacionada à segurança alimentar das populações, à melhoria da saúde pública e à inclusão social (ZEEUW, 2004).

A agricultura urbana é desenvolvida de forma individual ou coletiva (Ibid) por cidadãos, geralmente de baixa renda, que tem como objetivo principal a produção de alimentos para consumo próprio, acontecendo em menor proporção a comercialização de excedentes ou a produção exclusiva para a venda (MOUGEOT, 2000; NUGENT, 2000).

Com base na localização e na área territorial ocupada pela atividade de cultivo, a Organização das Nações Unidas para a Agricultura e Alimentação definiu uma classificação para essas atividades. Os pequenos espaços de produção localizados em áreas urbanas com grande densidade de edificações são classificados como de agricultura intra-urbana (FAO, 1999). Os principais espaços agrícolas intra-urbanos são: hortas comunitárias, hortas domésticas privadas, viveiros de muda, coberturas de edificações e beiras de rodovias e ferrovias (ZEEUW, 2004). Já, áreas maiores de cultivo, próximas aos limites urbanos e que possuam como foco a comercialização da produção, são classificadas como áreas de agricultura peri-urbana (FAO, 1999).

Pesquisas revelam o aumento do número de espaços destinados à atividade agrícola intra-urbana , apesar das altas densidades de edificações nas grandes cidades (p. ex. DEELSTRA; GIRARDET, 2000; NUGENT, 2000; ZEEUW, 2004). Deelstra e Girardet (2000) relatam que: Singapura produz $25 \%$ da sua demanda por vegetais; $67 \%$ das famílias de Dar-es-Salaam estão engajadas com a agricultura urbana; na cidade de Moscou, o número de habitantes envolvidos com produção urbana de alimentos aumentou de $20 \%$, em 1970 , para $65 \%$ em 1999; cerca de $50 \%$ dos vegetais consumidos em Havana são produzidos em hortas urbanas; $14 \%$ dos residentes de Londres e $44 \%$ dos habitantes de Vancouver produzem algum tipo de alimento nos seus quintais e, em Berlim, existem 80.000 
hortas comunitárias. Em cidades americanas, como Detroit e Nova Iorque, grandes áreas de terra tem sido disponibilizadas para pessoas desempregadas produzirem alimentos (REYNOLDS, 2010). Além disso, há um número significativo de iniciativas políticas para incentivar a implementação de áreas de agricultura urbana nas cidades (MENDES et al., 2008).

No Brasil, as experiências com agricultura urbana são verificadas, principalmente, nas capitais (SANTANDREU; LOVO, 2007). Pode-se destacar como exemplo o projeto de hortas comunitárias da região da Mooca, na cidade de São Paulo, o qual compreende 10,000 $\mathrm{m}^{2}$ de áreas cultivadas na região (Ibid.). A importância que esta atividade vem assumindo no país também é percebida através dos programas para o financiamento de projetos de hortas comunitárias criados pelo Ministério de Desenvolvimento Social (MDS, 2010).

A grande quantidade de experiências de agricultura urbana ao redor do mundo e a pressão política para a sua incorporação em processos de planejamento urbano evidenciam a importância e a escala que esta atividade vem assumindo. Parece haver, portanto, uma tendência para que espaços de cultivo se tornem cada vez mais presentes nos centros urbanos. Diante disso, parece importante considerar de que forma os espaços urbanos empregados para a produção de alimentos, são percebidos e utilizados, tanto por aqueles que os utilizam efetivamente, quanto por aqueles não envolvidos diretamente com o processo de cultivo, mas que convivem com esses espaços da cidade.

Levar em consideração esse aspecto parece ser fundamental na medida em que um indivíduo é afetado pelo ambiente e este é afetado pelo indivíduo (LANG, 1987). As características ambientais podem contribuir para atender, de maneira mais ou menos adequada, às necessidades fisiológicas, psicológicas e sociológicas dos indivíduos (Ibid.). Estes observam o ambiente em termos de suas demandas e, dessa forma, a compreensão do mesmo é amplamente baseada em suas necessidades e no que aprenderam a perceber (Ibid.). A priorização dessas necessidades pode variar, contudo, de acordo com a personalidade, a cultura e o nível sócio-econômico das pessoas (REIS, 1992).

Por outro lado, diferentes padrões do ambiente construído podem proporcionar diferentes comportamentos e experiências estéticas aos indivíduos (LANG, 1987). As propriedades visuais de um espaço podem produzir sentimentos positivos ou negativos, os quais, por sua vez, dependendo do estado psicológico do observador, o motivam a se aproximar ou a evitar determinado local (ULRICH, 1986). Portanto, a avaliação estética, favorável ou não, de um ambiente, influencia a experiência do usuário sobre o espaço (NASAR, 1997). Complementariamente, o espaço aberto público deve ter um uso adequado. Quando não utilizado ou utilizado por poucos, o espaço tende a ter pouco significado e importância, e a ser insatisfatório (REIS; LAY, 2006).

Entender de que maneira as características de determinado ambiente afetam seus usuários diretos e indiretos, é, portanto, fundamental para a avaliação da qualidade de um espaço urbano (Ibid.). As relações entre as características físico-espaciais de um ambiente e os indivíduos são estudadas pela área ambiente-comportamento com o objetivo de fornecer subsídios para o desenvolvimento de espaços que atendam adequadamente as necessidades humanas (Ibid.).

$\mathrm{Na}$ medida em que espaços de cultivo vêm se tornando mais presentes nas cidades, torna-se necessário abordar a relação entre esses espaços e os seus usuários. Ao associar o aumento de áreas verdes nas cidades com a qualificação do espaço urbano, a literatura sobre agricultura urbana aponta as atividades de cultivo como um meio para o aumento da qualidade de vida nas cidades (DEELSTRA; GIRARDET, 2000). Contudo, ainda se faz necessário um aprofundamento do conhecimento sobre a percepção de usuários diretos e indiretos das áreas de produção de alimento. Esta discussão parece ser relevante, principalmente, para as áreas de produção intra-urbanas. Diferentemente das áreas de agricultura peri-urbana, que se encontram em zonas de baixa densidade de ocupação, distantes dos centros urbanos, as áreas de agricultura intra-urbana estão inseridas em zonas de maior densidade de construções e de pessoas, gerando um maior contraste urbano e uma exposição a um número maior de indivíduos. 
As hortas comunitárias e as hortas em quintais privados são os espaços mais comuns de agricultura intra-urbana (FAO, 1999). As hortas comunitárias, por possuírem uma escala maior e permitirem o desenvolvimento de relações sociais, diferentemente das hortas em jardins privados, apresentam maior riqueza de aspectos a serem exploradas sob a perspectiva de uso e da percepção dos usuários.

Desse modo, tendo como foco as hortas comunitárias, o presente artigo tem como objetivo discutir a importância das áreas de agricultura intra-urbana a partir da abordagem da área de estudos ambiente-comportamento. É examinado de que forma usuários diretos e indiretos percebem e utilizam as hortas comunitárias, assim como o tipo de interação do usuário com o espaço, as relações sociais desenvolvidas nesses locais e as características visuais presentes nestes espaços. Essa discussão pretende oferecer elementos para se pensar o papel das hortas comunitárias frente às necessidades dos indivíduos e contribuir para um projeto mais qualificado desses locais.

\section{CARACTERIZAÇÃO DE HORTAS COMUNITÁRIAS}

As hortas comunitárias podem apresentar tamanhos variados, sendo utilizadas predominantemente para o cultivo de alimentos (LAWSON, 2004). Esses espaços podem ser manejados por poucas famílias ou então, quando ocupam áreas maiores, ser utilizados por um grande número de pessoas (Ibid.). Seus usuários são de diferentes faixas etárias e predomina a presença de mulheres (SANTANDREU; LOVO, 2007; ZEEUW, 2004; SHINEW et al., 2004). A maioria busca produzir para consumo próprio e com isso economizar em seu orçamento alimentar (NUGENT, 2000; LAWSON, 2004; SANTANDREU; LOVO, 2007). Outras vezes, vêem a atividade como um meio de complementar a renda familiar, a partir da venda de excedentes e, mais raramente, produzem exclusivamente para comercialização (NUGENT, 2000).

As hortas comunitárias estão, em geral, localizadas em bairros de baixa renda e em zonas residenciais revitalizadas da cidade (LAWSON, 2004). Esses espaços têm início, em grande parte, a partir de iniciativas de comunidades locais ou de incentivos do governo e de organizações não-governamentais (REYNOLDS, 2010). Estas atividades localizam-se, predominantemente, em áreas urbanas subutilizadas, de propriedade municipal, institucional ou privada, utilizadas sob a forma de concessão de uso (Ibid).

O cultivo de alimentos nas hortas comunitárias requer o intenso envolvimento de seus usuários, assim, o espaço assume características espaciais e de funcionamento que o fazem ser percebido ao mesmo tempo como um catalisador social e como um espaço privado (LAWSON, 2004). Dessa forma, esses locais apresentam aspectos peculiares do uso, como por exemplo: a associação do local com objetivos específicos de um grupo de usuários, a necessidade de responsáveis para a sua manutenção e a participação ativa de um grupo para o manejo do local (LAWSON, 2004). Por esse motivo, as hortas comunitárias não são classificadas como espaços públicos, por exemplo, como o são os parques (FRANCIS, 1989).

\section{INTERAÇÃO COM A NATUREZA EM HORTAS COMUNITÁRIAS}

A interação com a natureza é um dos motivos por que as pessoas utilizam áreas verdes públicas (CARR et al, 1992), interação essa reconhecida como benéfica e fundamental para o conforto psicológico do indivíduo (p. ex. KAPLAN, 1983; ULRICH, 1986, COOPER MARCUS; BARNES, 1998). Evidência da associação entre bem-estar e contato com a natureza são encontradas, por exemplo, em estudos que indicam que o contato com um ambiente natural desperta a atenção involuntária e contribui para a redução de estresse dos indivíduos (BERMAN et al., 2008) e que demonstram que vistas para espaços verdes em hospitais auxilia na recuperação de pacientes (ULRICH, 1986). 
No entanto, a experiência com a natureza em um espaço de cultivo apresenta características particulares. Nesses espaços a natureza é um componente essencial e não pode ser ignorada pelos usuários (KAPLAN, 1973), que são sensibilizados não só visualmente ou olfativamente, mas também através do tato como decorrência da manipulação da vegetação. Francis e Hester (1990) descrevem o jardim como um espaço de ação que requer envolvimento direto e íntimo e que proporciona relaxamento e alívio das tarefas diárias. Desse modo, lidar com a terra produz efeitos terapêuticos, pois reduz o estresse e contribui para o bem estar psicológico (FRANCIS; HESTER, 1990). Apesar de uma horta e um jardim apresentarem finalidades diversas, a interação requerida com o espaço é semelhante. O uso de jardins, produtivos ou não, gera bem estar, sentimentos de paz e tranquilidade e proporciona fascinação pelo ato de cultivar plantas (KAPLAN, 1983). Segundo Kaplan (1973), a fascinação é um componente que parece ser essencial para qualquer atividade considerada relaxante.

A jardinagem parece possuir muitas propriedades que tendem a elevar os níveis de fascinação. Primeiramente, ela se baseia nos processamentos básicos de informação que os seres humanos executam tão bem e pelos quais, presumidamente, zelam tanto. A jardinagem, não somente permite, como promove reconhecimento, previsão, controle e avaliação. O fazendo tanto através do fornecimento quanto da demanda por conhecimento. É um ambiente que permite a ordem, mas onde esta ordem está profundamente inserida na incerteza e na mudança. Portanto, ele desafia a capacidade humana de processamento de informações, e no momento em que o desafio é superado, gratificação e novos desafios emergem. Além disso, é uma atividade baseada na natureza, e a natureza por si tem sido um objeto de preferência em um nível impressionante. (KAPLAN, 1973, p. 160).

Adicionalmente, pesquisas desenvolvidas por Kaplan (1973) revelam os benefícios psicológicos gerados por jardins individuais, hortas coletivas e lotes para cultivo, aos seus usuários. Nessas pesquisas, todos os participantes relataram nível de satisfação semelhante na categoria de "escala de interesse continuado", a qual associava os jardins e as hortas a uma forma valiosa de gastar o tempo, obter distração da rotina e prazer estético, oportunizar relaxamento e proporcionar sensação de realização (Ibid). Para os usuários que produziam exclusivamente alimentos, como no caso das hortas comunitárias, a categoria de "benefícios tangíveis" (satisfação em produzir seu próprio alimento, economizar nas despesas de alimentação e colher a produção) foi também considerada uma importante fonte de satisfação (Ibid).

Outros estudos demonstram que, além dos benefícios obtidos a partir do trabalho com a terra, o envolvimento com hortas comunitárias é também motivado pela segurança para a realização de atividades ao ar livre e pela socialização proporcionada por esses espaços (SHINEW et al., 2004; KAPLAN, 1973; KINGSLEY et al., 2009). Além do cultivo de alimentos, algumas comunidades utilizam suas hortas coletivas para atender a outras demandas de usos, tais como áreas de recreação para crianças e áreas para descanso LAWSON, 2004). Portanto, as hortas comunitárias podem, além de proporcionar um contato ativo com a natureza, constituir espaços de socialização.

\section{HORTAS COMUNITÁRIAS, ESPAÇOS PARA A SOCIALIZAÇÃO}

O contato social motiva as pessoas a utilizarem os espaços públicos (CARR et al, 1992). Sentir-se seguro e parte de um grupo são necessidades humanas que podem ser atendidas a partir dos contatos sociais produzidos pelo uso dos espaços públicos (LANG, 1987). Em estudo realizado (REIS e LAY, 2010) em conjuntos habitacionais, ficou evidenciada a relação entre o uso de espaços comunais, o melhor relacionamento entre moradores e a maior satisfação em relação ao lugar de moradia, e, consequentemente, o papel desses espaços na geração de senso de comunidade.

Diversas pesquisas também demonstram que hortas comunitárias são percebidas por seus usuários como espaços de socialização, estimulando vizinhos a interagirem e a trabalharem em conjunto (p. ex. ARMSTRONG, 2000; KINGSLEY et al., 2009; SHINEW et al., 2004). Pesquisa realizada 
em hortas comunitárias de Nova Iorque (ARMSTRONG, 2000) mostra que a grande maioria dos seus usuários trabalhavam cooperativamente, dividindo as ferramentas, os vegetais produzidos e colaborando mutuamente nos processos de cultivo.

Esses resultados corroboram o destaque dado por Lewis (apud KAPLAN, 1983) à interação e cooperação entre as pessoas nas atividades de cultivo como um fator relevante para a geração de senso de comunidade. Ainda, foi constatado que a existência de hortas comunitárias nas vizinhanças analisadas contribuía para a melhoria da rede de relações sociais e para a capacidade de organização da comunidade local (ARMSTRONG, 2000).

O senso de comunidade, resultante da interação social, propicia o fortalecimento das comunidades, sendo fundamental para que o grupo se mobilize para reivindicar demandas e articular soluções próprias para os problemas locais (ARMSTRONG, 1993). Logo, conforme destaca Francis (1989), a presença de espaços comunais, como hortas comunitárias, cria oportunidades para que moradores estejam mais envolvidos com o desenvolvimento e com o controle de seu bairro. Zeeuw (2004) acrescenta que a implementação de hortas comunitárias, ao melhorarem esteticamente a vizinhança, transformando locais abandonadas em espaços verdes e produtivos, contribuem para o aumento da auto-estima e para a organização dos cidadãos, estimulando outras iniciativas na comunidade. As tarefas de cultivo e a oportunidade de socialização criada pelas hortas comunitárias também contribuem para que os usuários sintam-se parte da comunidade local e mais satisfeitos com o bairro onde vivem (KINGSLEY et al., 2009).

Evidência dos benefícios trazidos pelos espaços de cultivo coletivo para comunidades locais também é encontrada em pesquisas realizadas sobre agricultura urbana em capitais brasileiras. Essas pesquisas identificaram grande capacidade de auto-organização para reivindicação de demandas locais em comunidades envolvidas com atividades de cultivo (SANTANDREU; LOVO, 2007). Em geral, essas comunidades reivindicam melhores condições de infraestrutura e moradia, além de organizarem ações para a diminuição da criminalidade local (Ibid). A associação entre ações para o controle de criminalidade e presença de hortas comunitárias também foi verificada no estudo de Armstrong (2000), na cidade de Nova Iorque, onde o coordenador de uma horta comunitária relatou que a mesma promoveu um maior controle do bairro por meio da vigilância mútua realizada pelos moradores.

Percebe-se, portanto, que a interação social gerada pelas hortas comunitárias favorece a vigilância informal por parte dos residentes. Esse tipo de vigilância geralmente inicia quando os vizinhos começam a se conhecer (LAWSON, 2004). Para Jacobs (1961) a vigilância realizada de maneira informal pelos residentes e usuários de uma área, é uma estratégia efetiva para o aumento da segurança em um bairro. Esse aspecto é especialmente relevante para vizinhanças de baixa renda, as quais dependem, em grande parte, da vigilância realizada pelos próprios moradores, já que, em geral, não contam com uma segurança pública eficiente e não possuem recursos para implantar meios de controle privados (LAY, 1992).

Em função dos aspectos citados a agricultura urbana, de uma forma geral, e as hortas comunitárias, especificamente, vem sendo utilizadas para a revitalização de bairros de baixa renda em grandes cidades (ZEEUW, 2004). Por outro lado, como o sucesso de hortas comunitárias depende do grau de envolvimento dos usuários com o local, iniciativas do poder público para a revitalização de bairros degradados através da implementação de hortas comunitárias, tendem a não funcionar (LAWSON, 2004). Muitas vezes, a falta de adequação desses espaços frente às reais necessidades da comunidade local, resulta em áreas abandonadas e vandalizadas (Ibid.). Para que as hortas comunitárias se tornem espaços para a socialização de moradores, faz-se necessário que esses tenham interesse em se envolver com o espaço. Portanto, o projeto e o desenvolvimento das hortas deve ser uma iniciativa da comunidade ou ser realizado em conjunto com essa. 


\section{HORTAS COMUNITÁRIAS, ESPAÇOS TERRITORIALIZADOS}

As hortas comunitárias demandam um envolvimento contínuo e intenso de seus usuários diretos, representando muito mais um compromisso do que uma experiência casual com o espaço aberto. A reivindicação do uso do espaço é inerente a hortas comunitárias, pois para que o local atinja seu principal objetivo de produzir alimentos, a apropriação por um grupo que seja responsável por sua manutenção constante, faz-se necessária. Assim, observa-se que, em geral, as hortas comunitárias têm seu acesso restringido ao grupo responsável por sua manutenção (LAWSON, 2004). Nesse sentido, são espaços controlados e percebidos por usuários indiretos, como privados e territorializados (Ibid.). A percepção de controle, de privacidade e de territorialidade de hortas comunitárias está relacionada à reivindicação do uso do espaço por um grupo (PROSHANSKY et al. apud CARR et al., 1992). O controle está também relacionado, em geral, ao direito de uso de um determinado local por um grupo e a restrições sobre o seu acesso (CARR et al., 1992).

A limitação de acesso a um espaço pode ser estabelecida por meio de barreiras físicas, como grades, pela presença de vigilantes, pela falta de articulação com a circulação do entorno imediato ou pela visibilidade obstruída do local (CARR et al., 1992). Assim, embora as barreiras possam informar que o lugar pertence à alguém (KAPLAN et al., 1998), as mesmas limitam o uso do espaço pela população (CARR et al., 1992).

As hortas comunitárias são delimitadas, em sua maioria, por grades e sinalizadas por placas (ARMSTRONG, 2000). Geralmente, esses espaços são abertos ao público quando está presente algum membro do grupo responsável, permanecendo fechados no restante do tempo (Ibid.). A Figura 1, abaixo, representa situações de restrição de acesso a hortas comunitárias. Nas duas situações ilustradas, as hortas são delimitadas por cercas, o que informa que o local é controlado.

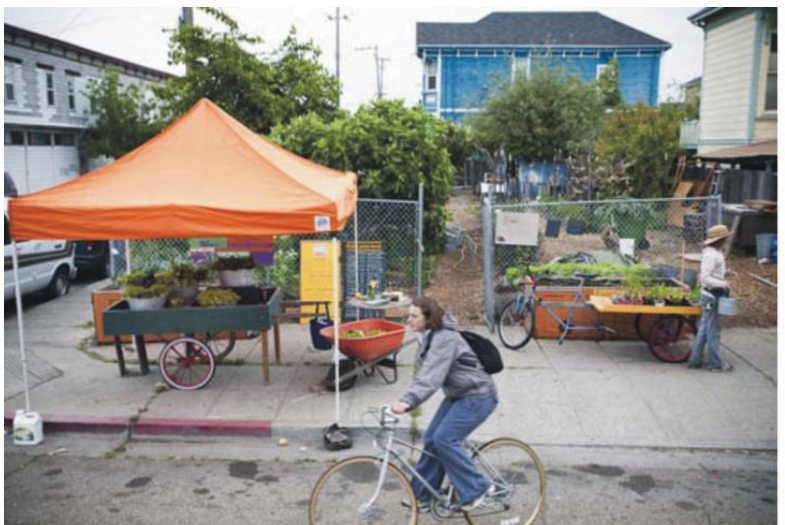

(A) Okland, Estados Unidos

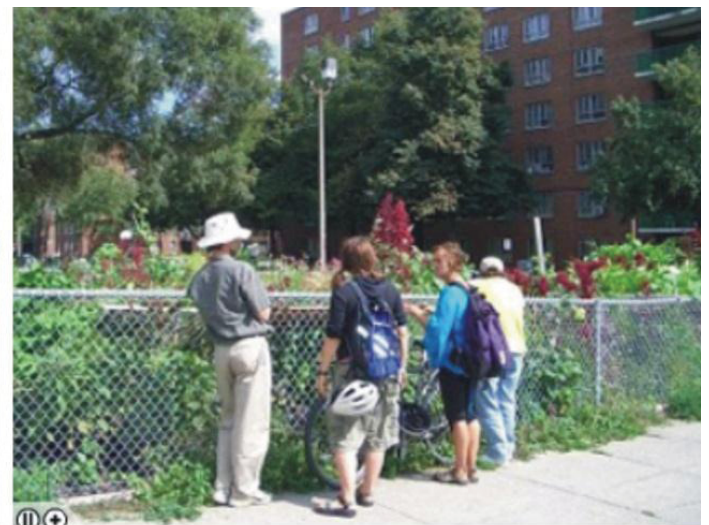

(B) Toronto, Canadá

Figura 1 - Exemplos de hortas comunitárias cercadas. Fonte: (A) CITY FARMER, 2010; (B) RUAF, 2010

De acordo com Carr et al. (1992), usuários indiretos tendem a respeitar e a valorizar um local percebido como controlado. Por outro lado, o estudo realizado por Armstrong (2000) sobre hortas comunitárias, indicou que a presença de barreiras físicas não garante, necessariamente, a segurança do local. A pesquisa demonstrou que, apesar de $67 \%$ das comunidades cercarem suas hortas, aproximadamente metade dos espaços analisados apresentou algum problema de vandalismo, sem diferença entre as ocorrências de vandalismo entre os espaços cercados e os não-cercados (Ibid.). Ao mesmo tempo, verificou-se, em outro estudo (LEWIS, 1972 apud KAPLAN, 1983), que o intenso envolvimento dos usuários com as hortas comunitárias contribui para a diminuição dos índices de vandalismo nesses locais, sugerindo que a presença de pessoas no espaço, e não a presença de barreiras físicas, parece ser o diferencial na redução do vandalismo. 
Apesar das hortas comunitárias serem, em geral, controladas, verifica-se que os usuários desses locais estimulam o engajamento de novos participantes (LAWSON, 2004). Na tentativa de tornar o espaço de hortas mais convidativo e incentivar seu uso pela população interessada, algumas hortas comunitárias dispõem de áreas demonstrativas, oferecem cursos e realizam feiras (Ibid.).

Além disso, é importante permitir que esses espaços possam ser freqüentados por um público amplo na medida em que eles proporcionam uma experiência com o ambiente natural que não está disponível em áreas verdes públicas. Segundo Alexander et al. (1977), para os habitantes das cidades que dependem exclusivamente de supermercados para atender sua demanda por alimentos, a falta de contato com a terra e o desconhecimento da origem dos alimentos consumidos, gera uma sensação de insegurança. Essa sensação poderia ser revertida na medida em que espaços produtivos estivessem mais acessíveis no ambiente urbano (Ibid.).

\section{CARACTERÍSTICAS VISUAIS DE HORTAS COMUNITÁRIAS}

Diversos estudos demonstram que cenas urbanas que incluem vegetação são preferidas em relação as que não contenham nenhum elemento natural (p. ex. ULRICH, 1986; REIS et al, 2010). A presença de vistas para elementos naturais contribui para a redução de sentimentos negativos relacionados às altas densidades urbanas (KEARNEY, 2006). Kaplan (1983) destaca ainda que a quantidade de elementos naturais que podem ser avistados pelas pessoas a partir de suas residências está associado ao nível de satisfação em relação ao local onde moram

No tocante ao cultivo de alimentos em área urbana, estudo realizado por Armstrong (2000) revelou que, na maioria dos bairros a presença de hortas comunitárias estimula atitudes mais positivas dos residentes em relação às suas vizinhanças, por meio do aumento na manutenção das áreas públicas e do plantio de árvores nas ruas, resultando na valorização dos terrenos do entorno. Bryld (2003) ressalta ainda, que as hortas comunitárias, em geral, contribuem positivamente para a melhoria do aspecto de bairros de baixa renda nas situações em que transformam terrenos baldios em áreas verdes cuidadas. No Brasil, por exemplo, empresas como a Eletrosul e a Eletropaulo, cujos terrenos sob as linhas de transmissão eram vandalizados, vêm cedendo esses espaços para as famílias, com moradias próximas produzirem alimentos e, assim, realizarem a manutenção dessas áreas (MDS, 2006). Parece haver, portanto, um impacto positivo em relação à percepção desses espaços nos casos em que eles recuperam terrenos urbanos anteriormente degradados.

No que diz respeito especificamente às características visuais de uma horta comunitária, percebe-se que a sua função resulta em uma configuração espacial recorrente com a presença de terrenos retangulares, conforme evidenciado mais adiante. Essas características afetam a percepção visual e as atitudes, já que os a mbientes são fontes de informação a serem processadas pelos usuários e determinados atributos físicos influenciam os níveis de preferência e satisfação do indivíduo (NASAR, 1997).

Kaplan et al. (1998) destacam que as pessoas tendem a preferir ambientes que sejam ao mesmo tempo envolventes (caracterizados por complexidade e mistério) e compreensíveis (caracterizados por coerência e legibilidade). A complexidade está ligada à riqueza e a variedade de elementos na paisagem, sendo que a pouca complexidade pode estar associada a um espaço monótono e a complexidade em demasia, a um espaço confuso, entendendo-se, nesse caso, complexidade como sinônimo de variação e não implicando em idéia de ordem. A dimensão do mistério diz respeito a atributos espaciais que instigam à exploração do espaço, como por exemplo, a presença de um caminho sinuoso ou de vegetação que esconda parcialmente o que está adiante. A coerência diz respeito a como o ambiente está organizado, e pode ser aumentada a partir da repetição de alguns aspectos ou pela presença de texturas que confiram unidade ao espaço. Por fim, a legibilidade corresponde à presença de elementos que auxiliam na compreensão e orientação espacial no ambiente (KAPLAN et al., 1998). 
Na prática, em cenas naturais, esses aspectos se traduzem na maior preferência por áreas verdes que combinem um terreno desobstruído com a presença de árvores mais espaçadas (Figura 2A), configurando uma paisagem com certo contraste e com acesso visual, sem a obstrução das vistas (KAPLAN, 1983). Por outro lado, cenas naturais com amplas áreas de cobertura vegetal não diferenciada (Figura 2B), ou seja, monótonas e de baixa complexidade, não oferecem maior estimulo visual, apresentando, assim, menores níveis de preferência (Ibid.). Paisagens desordenadas, de alta complexidade (ou grande variação), sem elementos focais, com vegetação muito densa obstruindo vistas (Figura 2C), dificultam a compreensão da cena, sendo também menos preferidas (Ibid.).

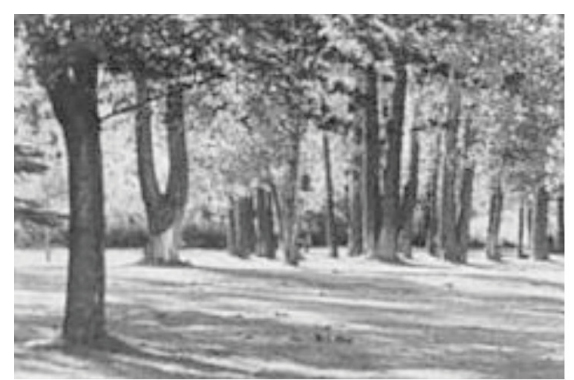

(A) Paisagem com acesso visual e sem obstrução de vistas

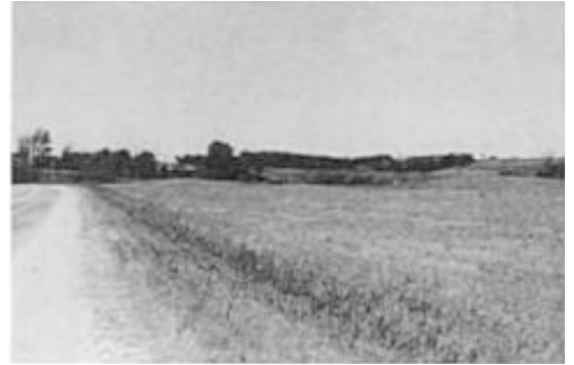

(B) Amplas áreas com cobertura vegetal não diferenciada

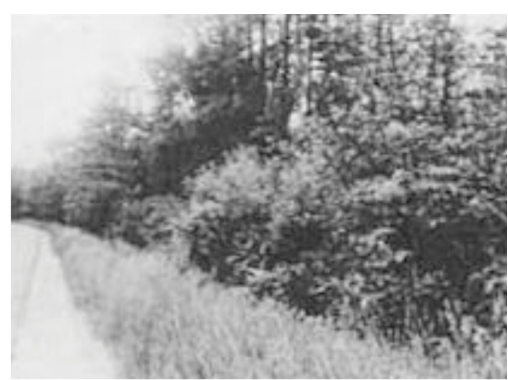

( C)Vegetação densa obstruindo : vista

Figura 2 - Exemplos de cenas naturais mais preferidas (A) e menos preferidas (B, C).

Fonte: KAPLAN et al., 1998, pp. 11,12

A consideração dos aspectos citados é fundamental para o projeto e a organização de áreas verdes (KAPLAN et al., 1998). Essas observações, mesmo que relacionadas a cenas naturais de uma forma geral, oferecem elementos para discutir a qualidade visual de hortas comunitárias. A Figura 3, abaixo, apresenta um grupo de imagens de hortas comunitárias em cidades dos Estados Unidos, em Cuba e no Brasil. As imagens foram selecionadas com base nos padrões de hortas comunitárias mais recorrentes, disponíveis em sítios sobre agricultura urbana.

Nas imagens da Figura 3, podem ser observadas semelhanças na organização dos elementos espaciais. Os espaços dessas hortas comunitárias são organizados a partir da disposição de canteiros retangulares para plantação. Os canteiros são dispostos ortogonalmente e separados por uma distância que permite apenas a circulação. A cena é de baixa complexidade e não há elementos que constituam a dimensão de mistério, aspectos que, conforme Kaplan et al. (1998), influenciam os níveis de preferência e satisfação do usuário no uso do espaço. 


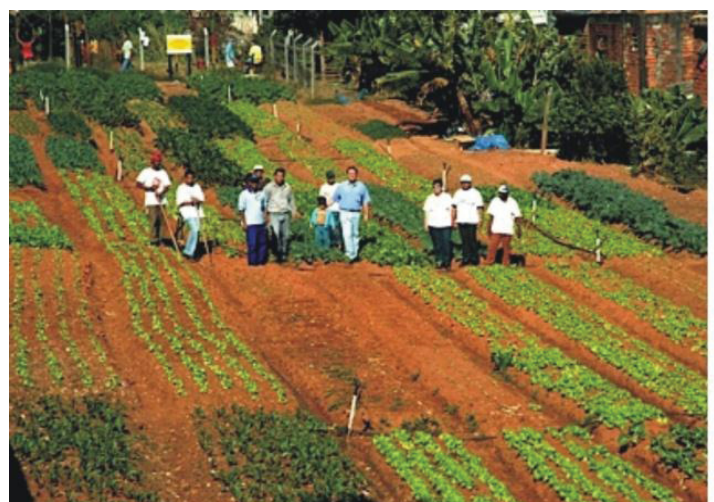

(A) São Paulo

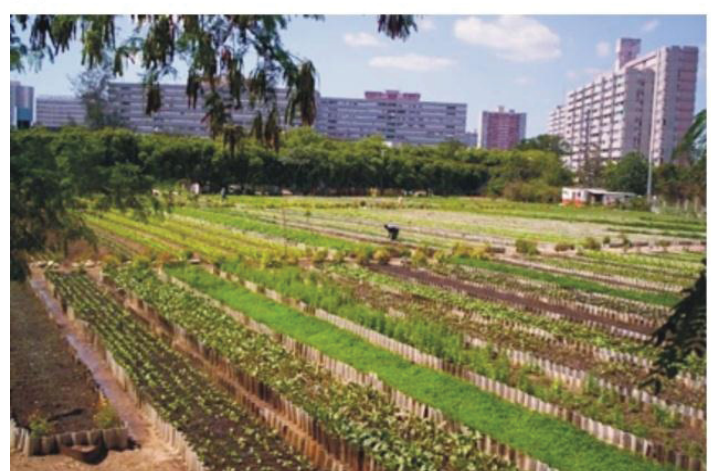

(C) Havana, Cuba

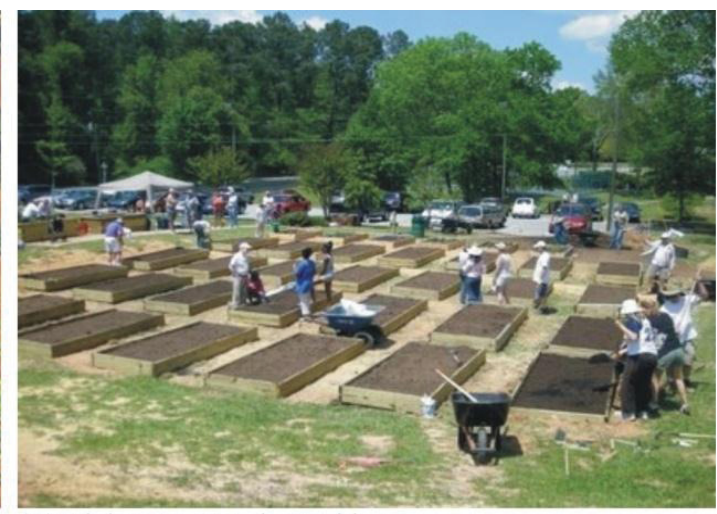

(B) Alpharetta, Estados Unidos

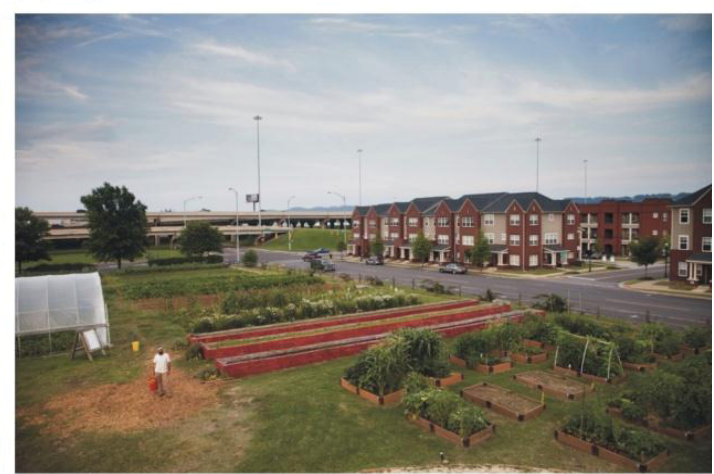

(D) Birmingham, Estados Unidos

Figura 3 - Imagens de hortas comunitárias em diversas cidades do mundo.

Porém, as características visuais das hortas comunitárias não precisam, necessariamente, estar restringidas por essa lógica de disposição dos canteiros, que está baseada na otimização do uso do espaço, no clima, na insolação e na topografia. Outros exemplos demonstram que, mesmo que o local seja destinado para a produção de alimentos, elementos podem ser introduzidos para aumentar o estímulo visual do ambiente (Figura 4).

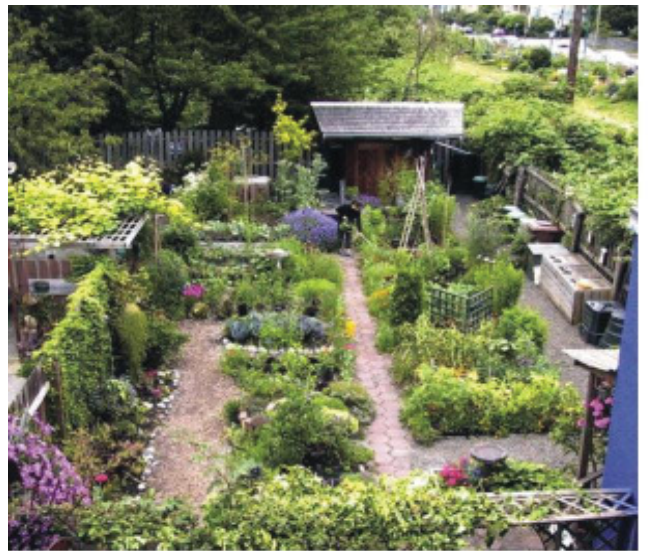

(A) Vancouver, Canadá

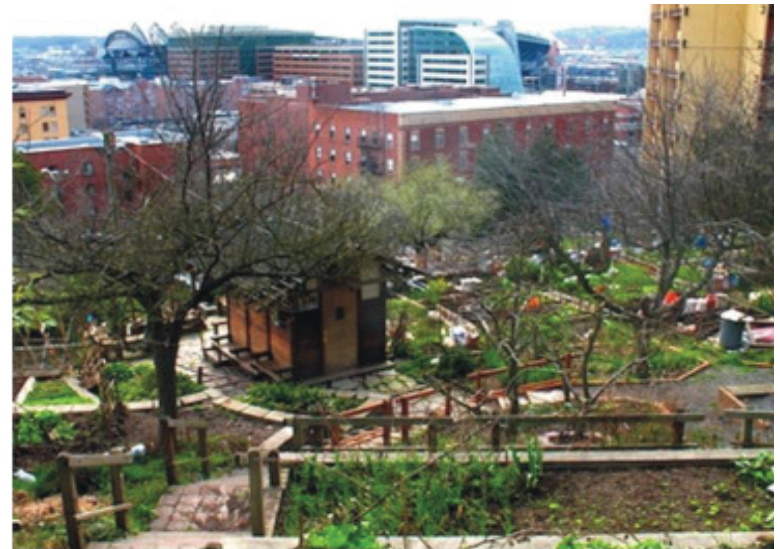

(B) Seattle, Estados Unidos

Figura 4 - Exemplos de hortas comunitárias que apresentam maior estimulo visual. Fonte: (A) RUAF , 2010; (B) CITY FARMER, 2010

Embora a Figura 4A também apresente hortas com áreas delimitadas por canteiros, a vegetação difere dos exemplos mostrados anteriormente (Figura 3). Já a Figura 4B, apresenta uma maior diversidade de elementos presentes na cena, incluindo árvores, com um conseqüente aumento da complexidade na cena. Contudo, estudos com base na avaliação dos níveis de satisfação e preferên- 
cia de usuários de hortas comunitárias que apresentem diferentes aspectos fisico-espaciais seriam necessários para explorar de maneira mais precisa o impacto exercido pela dimensão estética desses locais.

Cabe destacar ainda que as hortas comunitárias, apesar de sua configuração espacial característica, com a presença de canteiros e de grades (Figuras 1 e 3), também podem assumir configurações alternativas. A Figura 5, abaixo, traz duas imagens que representam casos em que a produção comunitária acontece ao longo de vias urbanas ou de linhas férreas. Nesses casos, não há a percepção de controle, pois os espaços não são cercados e o ambiente urbano é visualmente qualificado pela presença da vegetação.

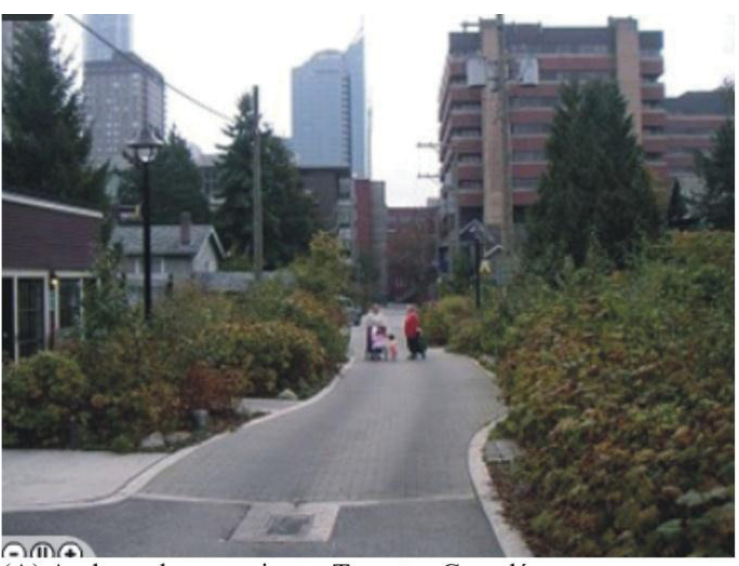

(A) Ao logo de uma via em Toronto, Canadá

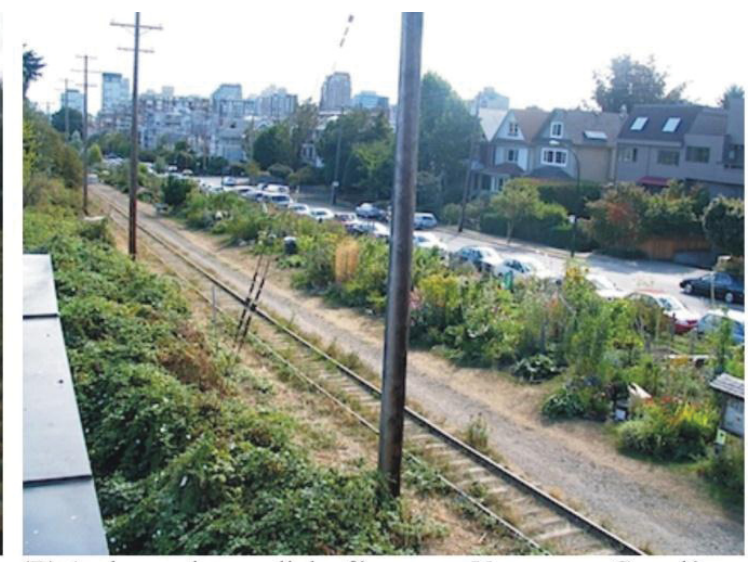

(B) Ao longo de uma linha férrea, em Vancouver, Canadá

Figura 5 - Localizações alternativas de hortas comunitárias.

Fonte: RUAF, 2010

\section{CONSIDERAÇÕES FINAIS}

A importância das áreas de agricultura intra-urbana a partir da abordagem da área de estudos ambiente-comportamento parece ter ficado evidenciada, assim como ficou salientada a relevância desta abordagem na discussão dos espaços de agricultura intra-urbana, ao considerar aspectos relacionados às percepções, atitudes e comportamentos dos usuários, diretos e indiretos, de hortas comunitárias.

As hortas comunitárias contribuem para o bem-estar psicológico de seus usuários diretos. $\mathrm{O}$ contato com a terra e a possibilidade de produzir o próprio alimento parecem constituir fontes de satisfação. Esses benefícios tendem a estar relacionados a todos os espaços de agricultura intra-urbana, uma vez que este tipo de interação é inerente a qualquer atividade de cultivo, seja em hortas domésticas, manejadas individualmente, cultivos realizados ao longo de ferrovias ou estradas, ou em coberturas de edificações.

As hortas comunitárias também são espaços para a socialização. Nesse sentido, estimulam a geração de senso de comunidade, contribuindo para a maior sensação de segurança na vizinhança e para a maior satisfação de seus usuários em relação ao local onde moram. A presença de hortas em comunidades também parece estar relacionada ao maior grau de manutenção de outros espaços abertos da vizinhança. Além disso, hortas comunitárias tendem a estimular a auto-organização da comunidade para a reivindicação de demandas sociais. Logo, a agricultura intra-urbana tende a desempenhar um papel importante para o fortalecimento de comunidades carentes, quando as hortas são manejadas coletivamente e desenvolvidas dentro destas comunidades.

Considerando esse potencial para a socialização, as hortas comunitárias que apresentam maior diversidade de uso, ou seja, que incluem outras atividades além da produção de alimentos, tendem a suprir demandas de uma maior parcela da comunidade. De acordo com Reis e Lay $(2006$, p.30) 
"a diversidade de atividades disponibilizadas aumenta a escolha, atraindo diferentes pessoas, em períodos diferentes, por razões variadas". Nesse sentido, flexibilizar as opções de uso em uma horta comunitária é um aspecto positivo que mereceria ser mais estimulado.

Também foi salientada a necessidade do intenso envolvimento e compromisso dos usuários das hortas comunitárias na sua manutenção, fazendo com que o espaço tenha determinadas características e seja percebido como territorializado e controlado. Assim, a incorporação de atividades de cultivo em áreas públicas, como parques, tenderia a ser afetada por tais características, o que sugere uma redução do uso de tais hortas. Contudo, estudos futuros poderiam analisar as percepções e comportamentos de usuários em relação a espaços de agricultura em parcelas de parques ou praças.

No que diz respeito à contribuição de hortas comunitárias para a qualificação estética do ambiente urbano, parece haver um efeito positivo nos casos em que esses locais revitalizam terrenos degradados. Contudo, as características visuais de uma horta comunitária, em geral, não incluem atributos espaciais que geram maior estímulo visual aos seus usuários. A comparação entre espaços de agricultura intra-urbana com diferentes características visuais e sua relação com os níveis de preferência e satisfação de usuários, diretos e indiretos, pode constituir uma abordagem para novos estudos. Ainda, tendo em vista que um espaço de cultivo de alimentos gera satisfações relacionadas à atividade de lidar com a terra, à interação com os ciclos naturais e ao consumo de alimentos produzidos localmente, comparar a importância da dimensão estética frente a esses benefícios, ou seja, analisar o grau de prioridade dos usuários em relação a esses aspectos, seria uma possibilidade para pesquisas adicionais.

Concluindo, o aprofundamento da discussão dos espaços de agricultura intra-urbana a partir da abordagem da área de estudos ambiente-comportamento auxilia na compreensão do papel desempenhado por esses espaços em relação às necessidades fisiológicas, psicológicas e sociais dos cidadãos e contribui para um projeto mais qualificado desses locais. O debate a partir dessa perspectiva ressalta também a importância de se pensar a incorporação dos espaços agrícolas intra-urbanos de uma forma integrada e harmônica no espaço urbano, de maneira a aumentar a qualidade estética das cidades.

\section{REFERÊNCIA BIBLIOGRÁFICA}

ALEXANDER, C.; ISHIKAWA, S.; SILVERSTEIN, M. A pattern language. New York: Oxford university Brooks, 1977.

ARMSTRONG, D. A survey of community gardens in upstate New York: Implications for health promotion and community development. Health \& Place, v. 6, p. 319-327, 2000.

ARMSTRONG, J. Making Community Involvement in Urban Regeneration Happen - Lessons from the United Kingdom. Community development journal, v. 28 n. 4, p.355-36, 1993.

BERMAN, M. G.; JONIDES, J.; KAPLAN, S. The Cognitive Benefits of Interacting with Nature. Psychological science, v. 19, n. 12, p. $1207-1211,2008$.

BRYLD, E. Potentials, Problems, and Policy Implications for Urban Agriculture in Developing Countries. Agriculture and human values, v. 20, p. 79-86, 2003.

CARR, S.; FRANCIS, M.; RIVLIN, L. G.; STONE, A. M. Public Space. New York: Cambridge University Press, 1992

CITY FARMER. Disponível em: <http://www.cityfarmer.info/>. Acessado em: 05 jun 2010.

DEELSTRA, T.; GIRARDET, H. Urban Agriculture and Sustainable Cities In: BAKKER, N. et al. (Ed.). Growing cities, growing food: urban agriculture on the policy agenda. DSE, 2000, p. 43-65.

FRANCIS, M. Urban Open Spaces, In: ZUBEN, E.; MOORE, G. (Ed.). Advances in environment, behavior and design. New York: Plenum Press, 1989. v. 1, p. 71-106.

FRANCIS, M.; HESTER, R. T. The meaning of gardens. London: The MIT Press, 1990. 
FOOD AND AGRICULTURE ORGANIZATION OF THE UNITED NATIONS - FAO. Issues in urban agriculture. 1999. Disponível em: < http://www.fao.org/ag/magazine/9901sp2.htm >. Acessado em: 15 jun/2010

JACOBS, J. (1961). Morte e vida de grandes cidades. São Paulo: Martins Fontes, 2009.

KAPLAN, R. Some Psychological Benefits of Gardening. Environment and behavior, v. 5, p. 145 - 162, 1973.

KAPLAN, R. The Role of Nature in the Urban Context, In: ALTMAN, I.; WOHLWILL, J. F. (Ed.). Behavior and the natural environment. New York: Plenum Press, 1983, p. $127-161$.

KAPLAN, R.; KAPLAN,S.; RYAN, R. L. With people in mind: design and management of everyday nature. Washington: Island Press, 1998.

KEARNEY, A. Residential development patterns and neighborhood satisfaction: impacts of density and nearby nature. Environment and behavior, v. 38, n. 1, p. 112-139, 2006.

KINGSLEY,J. Y.; TOWNSEND, M.; HENDERSON-WILSON, C. Cultivating health and wellbeing: members' perceptions of the health benefits of a Port Melbourne community garden. Leisure Studies, v. 28, n. 2, p. 207-219, 2009.

LANG, J. Fundamental processes of human behavior. In:

Creating architectural theory: The role of the behavioural sciences in environmental design. New York: Van Nostrand Reinhold Company, 1987. p. $84-98$.

LAY, M. C. Responsive site design, user environmental perception and behaviour. 1992. $290 \mathrm{f}$. Tese (Doctor of Philosophy em Arquitetura) - Post Graduate Research School, School of Architecture, Oxford Brookes University, Oxford, 1992.

LAWSON, L., The Planner in the Garden: A Historical View into the Relationship between Planning and Community Gardens. Journal of planning history, v. 3, n. 2, p. 151-176, 2004.

LEWIS, C. A. Public housing gardens - Landscapes for the soul. In: Landscape for living. Washington: USDA Yearbook of Agriculture, p. 277-282, 1972 apud KAPLAN, R. The Role of Nature in the Urban Context, In: ALTMAN, I.; WOHLWILL, J. F. (Ed.). Behavior and the natural environment. New York: Plenum Press, 1983, p. $127-161$.

MENDES, W.; BALMER, K., KAETHLER, T.; RHOADS, A. Using Land Inventories to Plan for Urban Agriculture Experiences from Portland and Vancouver. Journal of the American Planning Association, v. 74, n. 4 , p. $435-449,2008$.

MINISTÉRIO DO DESENVOLVIMENTO - MDS/BRASIL. Agricultura Urbana, 2010. Disponível em: $<$ http://www.mds.gov.br/falemds/perguntas-frequentes/seguranca-alimentar-e-nutricional/regiao-metropolitana/gestor/agricultura-urbana $>$. Acessado em: 18/06/2010.

MINISTÉRIO DO DESENVOLVIMENTO - MDS/BRASIL. Eletrosul beneficia 1.199 famílias com hortas comunitárias, 2006. Disponível em: < http://www.mds.gov.br/noticias/eletrosul-beneficia-1-199-familias-com-hortas-comunitarias/ >. Acessado em: 18/06/2010.

MOUGEOT, L.J.A. Urban Agriculture: Definition, Presence, Potentials and Risks. In: BAKKER, N. et al. (Ed.). Growing cities, growing food: urban agriculture on the policy agenda. DSE, 2000, p. 1-42.

NASAR, J. New Development in Aesthetics for Urban Design. In: MOORE, G.; MARANS, R. (Ed.). Advance in Environment Behavior and Design. vol. IV, Toward the Integration of Theory, Methods, Research, and Utilization. New York: Plenum Press, 1997.

NUGENT, R. The impact of urban agriculture on the household and local economies. In: BAKKER, N. et al. (Ed.). Growing cities, growing food: urban agriculture on the policy agenda. DSE, 2000, p. 67-97.

REIS, A. T. L. Mass housing, user participation and satisfaction. 1992. 290 f. Tese (Doctor of Philosophy in Architecture) - Post Graduate Research School, School of Architecture, Oxford Brookes University, Oxford, 1992.

REIS, A.; LAY, M. C. Avaliação da qualidade de projetos - uma abordagem perceptiva e cognitiva. Revista Ambiente Construído, Porto Alegre, v. 6, n. 3, p. 21-34, 2006.

REIS, A. T. L.; LAY, M. C. D. Internal and External Aesthetics of Housing Estates. Environment and Behavior, v. 42, n.2 p. 271 -294, 2010. 
REIS, A.; PEREIRA, M. L.; BIAVATTI, C. Percepção visual e impacto estético de vistas a partir de apartamentos. In: ENCONTRO NACIONAL, 13.; ENCONTRO LATINO-AMERICANO DE CONFORTO NO AMBIENTE CONSTRUÍDO, 2010, Canela. Anais... Canela: ANTAC, 2010.

REYNOLDS, K. A. Urban agriculture as revolution: an action research and social movement analysis of food production in Alameda County, California. 2010. $260 \mathrm{f}$. Tese (Doctor of Philosophy in Geography) Office of Graduate Studies, University of California, Davis, 2010.

RESOURCE CENTRES ON URBAN AGRICULTURE AND FOOD SECURITY - RUAF. Videos and slideshows on urban agriculture. Disponível em: < http://www.ruaf.org/node/1527>. Acessado em: 15 jun 2010.

SANTANDREU, A.; LOVO, I. C. Panorama da agricultura urbana e periurbana no brasil e diretrizes políticas para sua promoção: Identificação e Caracterização de Iniciativas de AUP em Regiões Metropolitanas Brasileiras. 2007. Disponível em: < http://www.rede-mg.org.br/article_get.php?id=100>. Acessado em: 23/05/2010.

SHINEW, K.J.; GLOVER, T. D.; PARRY, D. C. Leisure spaces as potential for interracial interaction: Community gardens in urban areas. Journal of leisure research, v. 36, n. 3, p. 336-355, 2004.

SMIT, J.; NASR, J. Urban agriculture for sustainable cities: using wastes and idle land and water bodies as resources. Environment and urbanization, v.4, p.141-152, 1992.

ULRICH, R. S. Human Responses to Vegetation and Landscapes. Landscape and Urban Planning, v.13, p.29-44, 1986.

ZEEUW, H. The development of urban agriculture, some lessons learnt. In: INTERNATIONAL CONFERENCE ON URBAN AGRICULTURE, AGRO-TOURISM AND CITY REGION DEVELOPMENT, 2004. Beijing. Key note paper.... Disponível em: < http://www.ruaf.org/node/1128>. Acessado em: 20/05/2010.

Trabalho enviado em novembro de 2011 Trabalho aceito em janeiro de 2012 